This paper is published as part of a Dalton Transactions themed issue on:

\title{
Supramolecular photochemistry
}

\author{
Guest Editors Michael D. Ward and Julia Weinstein \\ University of Sheffield, UK
}

Published in issue 20, 2009 of Dalton Transactions
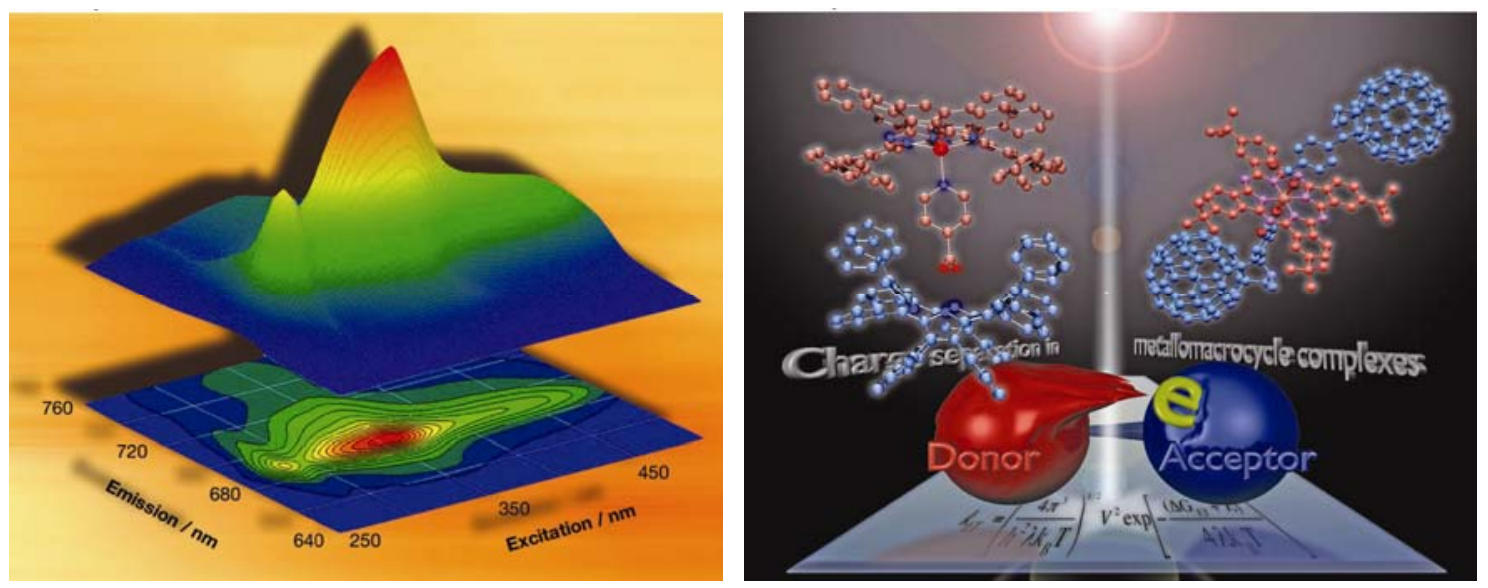

Images reproduced with permission of U. Nickel (left) and Shunichi Fukuzumi (right)

Papers published in this issue include:

Perspective: Sensitised luminescence in lanthanide containing arrays and $d-f$ hybrids

Stephen Faulkner, Louise S. Natrajan, William S. Perry and Daniel Sykes, Dalton Trans., 2009, DOI: $10.1039 / \mathrm{b} 902006 \mathrm{c}$

[Pt(mesBIAN)(tda)]: A near-infrared emitter and singlet oxygen sensitizer

Aaron A. Rachford, Fei Hua, Christopher J. Adams and Felix N. Castellano, Dalton Trans., 2009, DOI: $10.1039 / \mathrm{b} 818177 \mathrm{~b}$

Self-assembly of double-decker cages induced by coordination of perylene bisimide with a trimeric Zn porphyrin: study of the electron transfer dynamics between the two photoactive components Ana I. Oliva, Barbara Ventura, Frank Würthner, Amaya Camara-Campos, Christopher A. Hunter, Pablo Ballester and Lucia Flamigni, Dalton Trans., 2009, DOI: 10.1039/b819496c

Synthesis, photophysical and electrochemical characterization of phthalocyanine-based poly $(p-$ phenylenevinylene) oligomers

Juan-José Cid, Christian Ehli, Carmen Atienza-Castellanos, Andreas Gouloumis, Eva-María Maya, Purificación Vázquez, Tomás Torres and Dirk M. Guldi, Dalton Trans., 2009, DOI: $\underline{10.1039 / \mathrm{b} 818772 \mathrm{j}}$

Luminescence, electrochemistry and host-quest properties of dinuclear platinum(II) terpyridyl complexes of sulfur-containing bridging ligands

Rowena Pui-Ling Tang, Keith Man-Chung Wong, Nianyong Zhu and Vivian Wing-Wah Yam, Dalton Trans., 2009, DOI: 10.1039/b821264c

Visit the Dalton Transactions website for more cutting-edge inorganic and organometallic research www.rsc.org/dalton 


\title{
Multichromophoric supramolecular systems. Recovery of the distributions of decay times from the fluorescence decays
}

\author{
Vincent Souchon, ${ }^{a}$ Isabelle Leray, ${ }^{a}$ Mário N. Berberan-Santos ${ }^{* c}$ and Bernard Valeur ${ }^{* a, b}$ \\ Received 6th January 2009, Accepted 17th February 2009 \\ First published as an Advance Article on the web 13th March 2009 \\ DOI: 10.1039/b900263b
}

The fluorescence decays of a tetrachromophoric calix[4]arene and a heptachromophoric cyclodextrin with appended pyridin-2'-yl-1,2,3-triazole groups, and their complexes with the cadmium ion, are analyzed with a sum of compressed hyperbola. Such functions are shown to be well suited for the recovery of the underlying distributions of decay times. The heterogeneity of the micro-environments experienced by the chromophores, and/or their mutual interactions in the excited state can in this way be displayed graphically. A fit with a sum of four exponentials is also found to be satisfactory, but the respective parameters lack direct physical meaning.

\section{Introduction}

Various multi-chromophoric supramolecular systems have been conceived for light harvesting, ${ }^{1}$ for ion and molecule recognition, ${ }^{2}$ and for performing specific functions in supramolecular devices. ${ }^{3}$ Most of these systems exhibit complex fluorescence spectra and decays as a result of the interactions between chromophores in the excited state at short distances (e.g. excimer formation and photoinduced electron transfer) or at long distances (Förster resonance energy transfer (FRET)). Moreover, the chromophores may experience different micro-environments that affect the rate of their spontaneous decay.

The fluorescence decays of multi-chromophoric systems are most often analyzed with a sum of exponentials: the number of terms is increased until a satisfactory fit is obtained, up to a practical limit of four exponentials. In most cases, it is difficult to assign a physical significance to the decay times or rate constants. In fact, in many cases, the flexibility of the links between the chromophores and the molecular skeleton or platform leads one to expect a distribution of rate constants owing to the distribution of inter-chromophoric distances and angles and/or the microheterogeneity of the chromophore environments.

Recovery of a distribution from an experimental fluorescence decay is very difficult because this is an ill-conditioned problem. ${ }^{4}$ In the absence of a physical model, the best approach would be in principle the use of methods without assumption of the distribution shape such as the maximum entropy method ${ }^{5}$ and the exponential series method. ${ }^{6}$ However, these methods are extremely sensitive to data quality and truncation effects. Therefore, it is preferable to use mathematical functions whose choice is $a$ posteriori validated by a good fit with a small number of floating parameters. In this regard, it was shown by two of $\mathrm{us}^{4}$ that two

anstitut d'Alembert, Laboratoire PPSM (CNRS UMR 8531), ENSCachan, 61 Av. du Président Wilson, F-94235, Cachan Cedex, France ${ }^{b}$ Département Sciences et Techniques Industrielles, Conservatoire National des Arts et Métiers, 292 rue Saint-Martin, F-75141, Paris Cedex 03, France. E-mail: bernard.valeur@cnam.fr

${ }^{c}$ CQFM - Centro de Química-Física Molecular and IN-Institute of Nanoscience and Nanotechnology, Instituto Superior Técnico, Universidade Técnica de Lisboa, 1049-001, Lisboa, Portugal.E-mail: berberan@ist.utl.pt functions are very well suited to the analysis of luminescence decays with underlying distributions: the well-known stretched exponential (or Kohlrausch) function, ${ }^{4 b}$

$$
I(t)=\exp \left[-\left(t / \tau_{0}\right)^{\beta}\right]
$$

where $0<\beta<1$, and the less-known compressed hyperbola (or Becquerel) function, ${ }^{4 c}$

$$
I(t)=\frac{1}{\left(1+c t / \tau_{0}\right)^{1 / c}}
$$

where $0<c<1$. The parameter $\tau_{0}$ has dimensions of time in both cases.

The aim of this work is to explore the potentialities of compressed hyperbola to recover the underlying distributions of rate constants from the fluorescence decays of two multi-chromophoric systems: a tetrachromophoric calix[4]arene (Calix-PT4) and a heptachromophoric $\beta$-cyclodextrin ( $\beta$-CD-PT7) with appended pyridin-2'-yl-1,2,3-triazole groups (Scheme 1). The synthesis and photophysics of these systems have been previously reported. ${ }^{7}$ In the present paper, special attention is paid to the fluorescence decays of the complexes of these systems with cadmium(II).
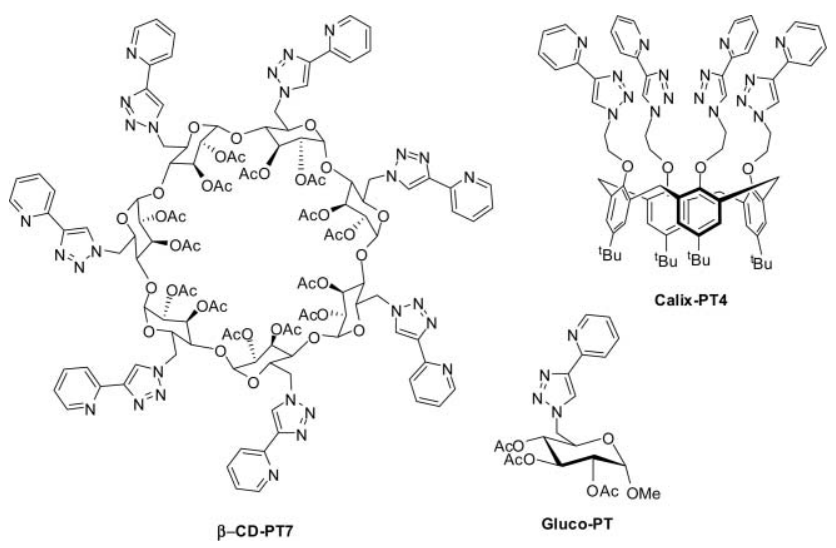

Scheme 1 Structures of the tetrachromophoric calix[4]arene (Calix-PT4) and the heptachromophoric $\beta$-cyclodextrin ( $\beta$-CD-PT7) with appended pyridin-2'-yl-1,2,3-triazole groups. Gluco-PT is the model chromophore. 


\section{Results and discussion}

\section{Description of complex fluorescence decays with distributions}

For completely monotonic decays, the fluorescence intensity $I(t)$ is given by the integrals: ${ }^{4 a}$

$$
I(t)=\int_{0}^{\infty} f(\tau) \exp (-t / \tau) \mathrm{d} \tau=\int_{0}^{\infty} g(k) \exp (-k t) \mathrm{d} k
$$

where $f(\tau)$ and $g(k)$ are the probability density functions (pdf) of lifetimes and rate constants, respectively.

For the Becquerel decay function, $g(k)$ is the gamma pdf, given by: ${ }^{4 a}$

$$
g(k)=\frac{\alpha}{\Gamma(1 / c)}(\alpha k)^{\frac{1}{c}-1} \exp (-\alpha k)
$$

with $\alpha=\tau_{0} / c$. The analogous probability density function of lifetimes $f(\tau)$ is easily shown to be given by:

$$
f(\tau)=\frac{1}{\alpha \Gamma(1 / c)}\left(\frac{\alpha}{\tau}\right)^{\frac{1}{c}+1} \exp \left(-\frac{\alpha}{\tau}\right)
$$

This function is known as the inverse-gamma pdf. Eqn (4) and (5) are flexible distribution functions because they can closely approximate the actual distribution in many situations ranging from very narrow distributions $(c \approx 0)$, where the decay is essentially exponential, to very broad $(c \approx 1)$ distributions, where the decay is close to hyperbolic, with a power law dependence on time (for sufficiently long times).

The distribution function eqn (5) is asymmetric for any $c>0$ and its maximum occurs at $\tau_{0} /(1+c)$, whereas the ensemble average decay time is $\langle\tau\rangle=\int_{0}^{\infty} \tau f(\tau) d \tau=\int_{0}^{\infty} I(t) d t=\frac{\tau_{0}}{1-c}$. This ensemble average differs from the average decay time $\mathrm{tab}^{\mathrm{b}} \frac{\int_{\tau}^{\infty} t I(t) d t}{\int_{0}^{\infty} I(t) d t}=\frac{\left\langle\tau^{2}\right\rangle}{\langle\tau\rangle}$, which is finite only for $c<\frac{1}{2}$, and is in that case given by $\bar{\tau}=\frac{\tau_{0}}{1-2 c}$.

A still more general approach is to write a complex decay as a sum of Becquerel (compressed hyperbola) functions,

$$
I(t)=\sum_{i} \frac{a_{i}}{\left(1+c_{i} t / \tau_{i}\right)^{1 / c_{i}}}
$$

with $\sum a_{i}=1$. We assume here that all $a_{i}$ are positive. The distribution of lifetimes corresponding to eqn (6) is a weighted sum of Becquerel pdfs:

$$
f(\tau)=\sum_{i} a_{i} f_{i}(\tau)=\sum_{i} \frac{a_{i}}{\alpha_{i} \Gamma\left(1 / c_{i}\right)}\left(\frac{\alpha_{i}}{\tau}\right)^{\frac{1}{c_{i}}+1} \exp \left(-\frac{\alpha_{i}}{\tau}\right)
$$

and the ensemble average decay time is thus:

$$
\langle\tau\rangle=\sum_{i} \frac{a_{i} \tau_{i}}{1-c_{i}}
$$

\section{Formation of complexes upon binding of cadmium ion to Calix-PT4 and $\beta$-CD-PT7}

We previously showed that the photophysical properties of CalixPT4 and $\beta$-CD-PT7 were strongly affected by the complexation with $\mathrm{Cd}^{2+}$ in a very different way. ${ }^{7}$ For Calix-PT4, the appearance of a new band at $480 \mathrm{~nm}$ led us to conclude that excimer formation is induced by cadmium binding, whereas no excimer was formed upon binding of cadmium to $\beta$-CD-PT7 (Fig. 1). The absence of cation-induced excimer formation in $\beta$-CD-PT7 is likely to be due to the poorly flexible link between the pyridinyl triazole moieties and the rim, and to the rather long distance between the atoms to which these moieties are bound, so that the required conformation for excimer formation cannot be reached. In contrast, the shorter distances between attach points and the more flexible link in Calix-PT4 are much more favourable to excimer formation.
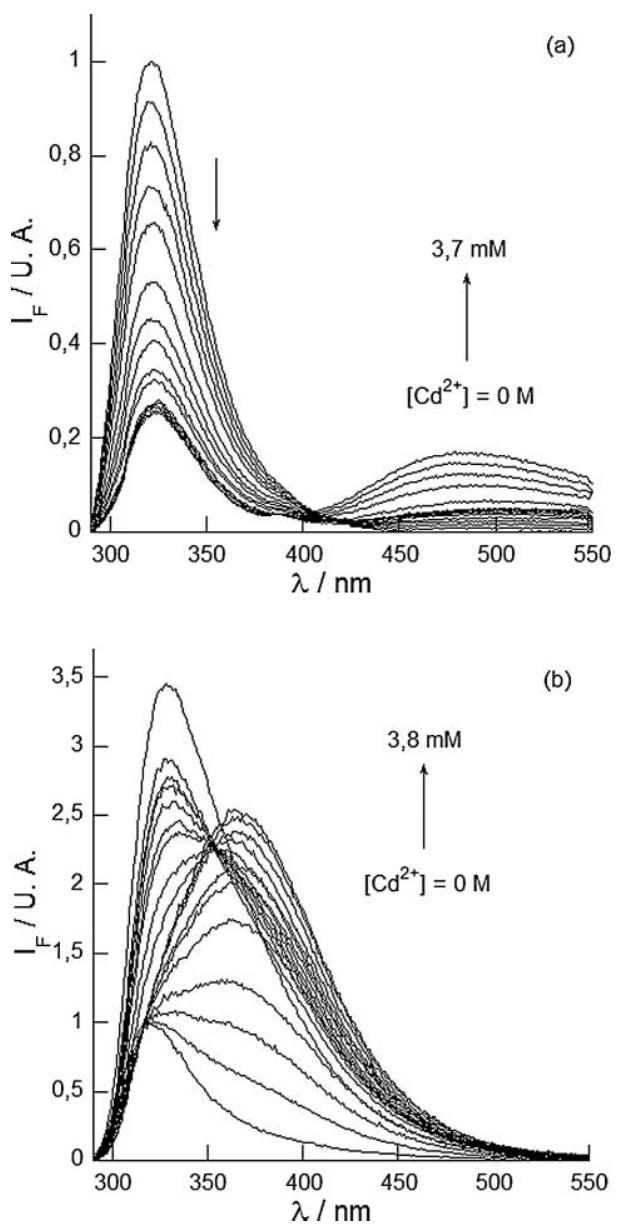

Fig. 1 Evolution of the fluorescence spectra upon addition of cadmium ion to a solution of Calix-PT4 (a) and $\beta$-CD-PT7 (b) in methanol (concentration: $8.8 \mathrm{mM}$; $\lambda_{\mathrm{exc}}=280 \mathrm{~nm}$ ) (From ref. 7).

Global analysis of the absorption or fluorescence spectra (by means of the SPECFIT software) at various concentrations of cadmium provided the stability constants of the complexes that are formed (Table 1). 
Table 1 Stability constants ${ }^{a}$ of the complexes of Calix-PT4 and $\beta-C D-$ PT7 with $\mathrm{Cd}^{2+}$ (From ref. 7)

\begin{tabular}{llll}
\hline Ligand & $\log \beta_{11}$ & $\log \beta_{21}$ & $\log \beta_{31}$ \\
\hline Calix-PT4 & $6.0 \pm 0.15$ & $8.7 \pm 0.15$ & - \\
$\beta$-CD-PT7 & $8.8 \pm 0.2$ & $13.9 \pm 0.3$ & $15.8 \pm 0.4$ \\
${ }^{a} \beta_{\mathrm{i} 1}=\frac{\left[\mathrm{M}_{\mathrm{i}} \mathrm{L}\right]}{[\mathrm{M}]^{\mathrm{i}}[\mathrm{L}]}$ & & & \\
\end{tabular}

Table 2 Data analysis of the fluorescence decays of Calix-PT4 $(4.2 \mu \mathrm{M})$ in methanol in the absence and in the presence of an excess of cadmium $\left(\left[\mathrm{Cd}^{2+}\right]=16 \mathrm{mM}\right)$. Excitation wavelength: $288 \mathrm{~nm}$. The model is a sum of exponentials with decay times $\tau_{\mathrm{i}}$, pre-exponential factors $\alpha_{\mathrm{i}}$ and fractional intensities $f_{\mathrm{i}}$ defined as: $f_{i}=\frac{\alpha_{i} \tau_{i}}{\sum \alpha_{i} \tau_{i}}$

\begin{tabular}{lclll}
\hline & $\tau_{\mathrm{i}} / \mathrm{ns}$ & $\alpha_{\mathrm{i}}$ & $f_{\mathrm{i}}$ & $\chi_{R}{ }^{2}$ \\
\hline Calix-PT4 $^{a}$ & $0.033 \pm 0.002$ & 0.710 & 0.34 & 1.11 \\
& $0.116 \pm 0.005$ & 0.273 & 0.45 & \\
& $0.54 \pm 0.06$ & 0.015 & 0.12 & \\
$2 \mathrm{Cd}^{2+} \subset$ Calix-PT4 $^{b}$ & $4.4 \pm 1.3$ & 0.002 & 0.09 & \\
& $0.012 \pm 0.001$ & 0.995 & 0.73 & 1.32 \\
& $0.48 \pm 0.07$ & 0.004 & 0.13 & \\
$\mathrm{CCd}^{2+} \subset$ Calix-PT4 $^{c}$ & $4.3 \pm 1.3$ & 0.001 & 0.14 & \\
& $0.050 \pm 0.02$ & 0.42 & 0.01 & 1.22 \\
& $0.64 \pm 0.12$ & 0.07 & 0.02 & \\
& $3.88 \pm 0.07$ & 0.34 & 0.49 & \\
& $7.3 \pm 0.12$ & 0.17 & 0.48 &
\end{tabular}

${ }^{a}$ Observation at $330 \mathrm{~nm}$. Channel width: $14.6 \mathrm{ps} .{ }^{b}$ Observation at $330 \mathrm{~nm}$. Channel width: 7.32 ps. ${ }^{c}$ Observation at $480 \mathrm{~nm}$. Channel width: $39 \mathrm{ps}$.

\section{Fluorescence decays of Calix-PT4 and its complexes with $\mathrm{Cd}^{2+}$}

Analysis of the fluorescence decay of Calix-PT4 with a sum of exponentials led us to conclude that four exponential terms were necessary to get a satisfactory fit (see Table 2). Then, we tried to fit the decay with compressed hyperbola. It turned out that a sum of two compressed hyperbola gave a very good fit with a chi-squared value of $1.15 .{ }^{7}$ However, the relative amplitude of the second hyperbola was only $3 \%$ and the shape of the recovered distribution was thus only slightly affected when going from one to two components. This observation showed that there is no doubt on the existence of a distribution of decay times - or rate constants - and that the four discrete decay times have no physical meaning; they are only fitting parameters. Such a distribution can be accounted for by the heterogeneity of the micro-environments of the chromophores.

Let us examine now the fluorescence decay in the presence of an excess of $\mathrm{Cd}^{2+}$ (Fig. 2). Since the evolution of the fluorescence spectra can be interpreted by cation-induced formation of excimers, we looked for a rise time decay recorded at $480 \mathrm{~nm}$, i.e. at the wavelength corresponding to the intensity maximum of the excimer band. However, an analysis with a sum of exponentials did not reveal any rise time (Table 2). Considering that the shortest decay time that can be measured with our instrument is about $10 \mathrm{ps}$, the absence of detected rise time means that the excimers are preformed.

In Table 3 are reported the results of the analysis of the fluorescence decays with a sum of two compressed hyperbola.
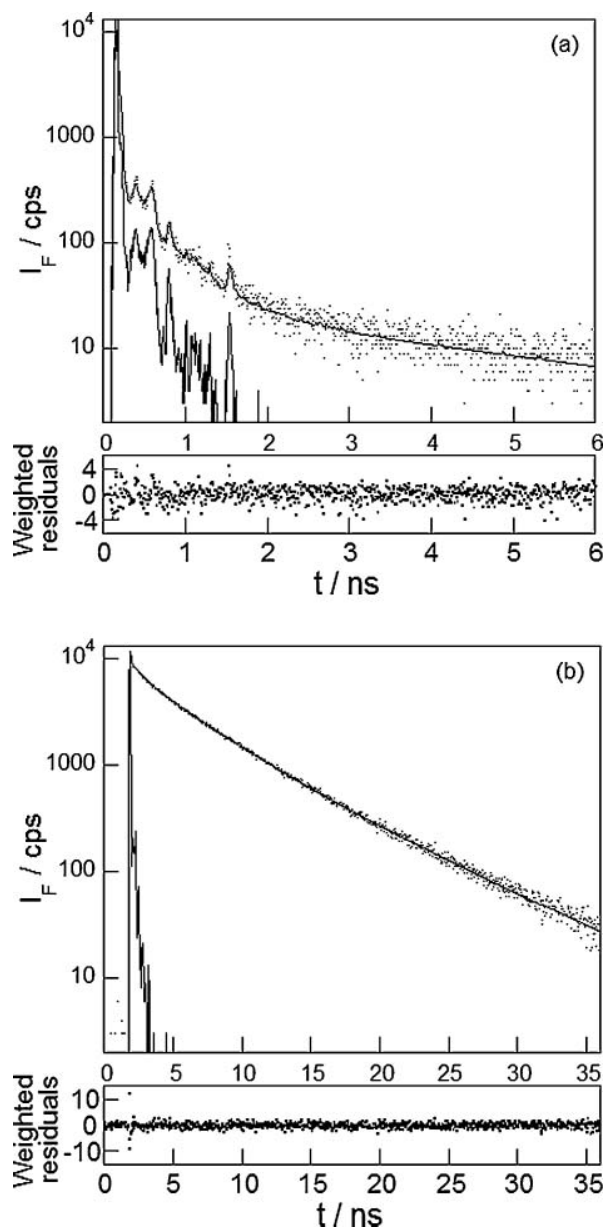

Fig. 2 Fluorescence decays of Calix-PT4 $(4.2 \mu \mathrm{M})$ in methanol in the presence of an excess of cadmium $\left(\left[\mathrm{Cd}^{2+}\right]=16 \mathrm{mM}\right)$. Excitation wavelength: $288 \mathrm{~nm}$. Observation wavelength: (a) $330 \mathrm{~nm}$; (b) $480 \mathrm{~nm}$. Channel width: 7.32 ps. The solid line represents the best fit with a sum of four exponentials.

Table 3 Data analysis of the fluorescence decays of Calix-PT4 $(4.2 \mu \mathrm{M})$ in methanol in the absence and in the presence of an excess of cadmium $\left(\left[\mathrm{Cd}^{2+}\right]=16 \mathrm{mM}\right)$. Excitation wavelength: $288 \mathrm{~nm}$. The model is a sum of compressed hyperbola according to eqn (3). $x_{\mathrm{i}}$ are the fractional intensities defined as: $x_{i}=\frac{a_{i} \tau_{i} /\left(1-c_{i}\right)}{\sum_{i} a_{i} \tau_{i} /\left(1-c_{i}\right)}$

\begin{tabular}{|c|c|c|c|c|c|}
\hline & $\tau_{\mathrm{i}} / \mathrm{ns}$ & $c_{\mathrm{i}}$ & $a_{\mathrm{i}}$ & $x_{\mathrm{i}}$ & $\chi_{R}^{2}$ \\
\hline \multirow[t]{2}{*}{ Calix-PT4 ${ }^{a}$} & 0.042 & 0.34 & 0.97 & 0.49 & \multirow[t]{2}{*}{1.16} \\
\hline & 0.097 & 0.96 & 0.03 & 0.51 & \\
\hline \multirow{2}{*}{$2 \mathrm{Cd}^{2+} \subset$ Calix-PT$^{b}$} & 0.008 & 0.12 & 0.995 & 0.72 & \multirow[t]{2}{*}{1.89} \\
\hline & 0.36 & 0.60 & 0.005 & 0.28 & \\
\hline \multirow[t]{2}{*}{$2 \mathrm{Cd}^{2+} \subset$ Calix-PT$^{c}{ }^{c}$} & 0.19 & 0.60 & 0.56 & 0.07 & \multirow[t]{2}{*}{1.02} \\
\hline & 4.57 & 0.091 & 0.44 & 0.93 & \\
\hline
\end{tabular}

${ }^{a}$ Observation at $330 \mathrm{~nm}$. Channel width: 14.6 ps. ${ }^{b}$ Observation at $330 \mathrm{~nm}$. Channel width: 7.32 ps. ${ }^{c}$ Observation at $480 \mathrm{~nm}$. Channel width: 39 ps.

The recovered distributions of decay times are given in Fig. 3. In the absence of cadmium, the average decay time is $0.13 \mathrm{~ns}$, which is close to the lifetime of 0.11 ns measured for the model 


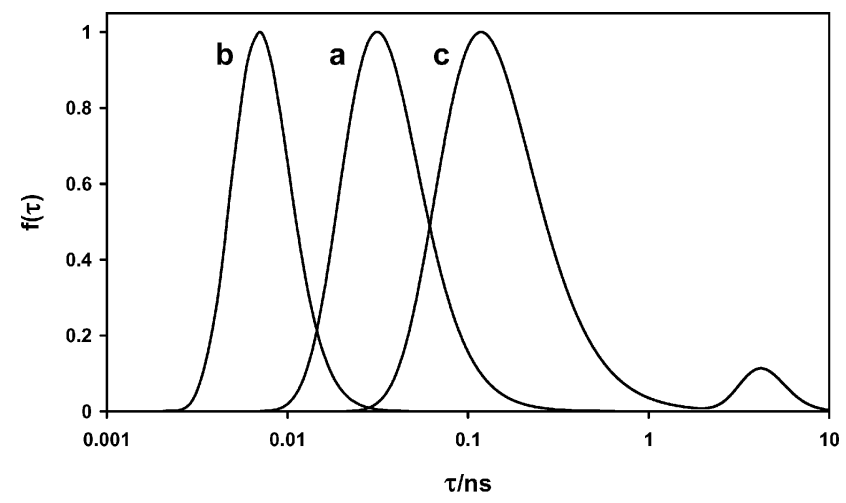

Fig. 3 Recovered distributions of decay times for Calix-PT4 $(4.2 \mu \mathrm{M})$ in methanol in the absence of cadmium (a) and in the presence of an excess of cadmium $\left(\left[\mathrm{Cd}^{2+}\right]=16 \mathrm{mM}\right)$ for an emission wavelength of $330 \mathrm{~nm}$ (b) and $480 \mathrm{~nm}$ (c). For displaying purposes, each distribution was normalized at the respective maximum.

chromophore (Gluco-PT) in methanol. ${ }^{7}$ In the presence of an excess of cadmium, the lifetime of the complex of Gluco-PT with $\mathrm{Cd}^{2+}$ is $0.55 \mathrm{~ns}$. The average decay time of the Calix-PT4 complex upon observation at $330 \mathrm{~nm}$ is $13 \mathrm{ps}$, i.e. much shorter than $0.55 \mathrm{~ns}$. In contrast, upon observation at $480 \mathrm{~nm}$ the CalixPT4 complex shows longer decay times: the average decay time is $3.0 \mathrm{~ns}$. These results are consistent with excimer formation induced by complexation with $\mathrm{Cd}^{2+}$. There is indeed an overall quenching of the monomer band (around $300 \mathrm{~nm}$ ) accompanied by a decrease of decay time, while an excimer band appears together with longer decay times.

The distributions shown in Fig. 3 provide a direct visualization of the heterogeneity of the micro-environments experienced by the chromophores, together with their mutual interactions in the excited state, i.e. excimer formation in the present case. The distributions are almost unimodal, except in the presence of cadmium when observing the fluorescence in the excimer band.

\section{Fluorescence decays of $\beta$-CD-PT7 and its complexes with $\mathbf{C d}^{2+}$}

As in the case of Calix-PT4, a sum of four exponentials gave a satisfactory fit for the decay of $\beta$-CD-PT7 in the presence and in the absence of cadmium (Table 4). The fluorescence decay in the presence of one equivalent of cadmium is shown in Fig. 4.

Table 4 Data analysis of the fluorescence decays of $\beta$-CD-PT7 $(1.7 \mu \mathrm{M})$ in methanol in the absence and in the presence of one equivalent of cadmium or in the presence of an excess of cadmium $\left(\left[\mathrm{Cd}^{2+}\right]=0.32 \mathrm{mM}\right)$. Excitation wavelength: $288 \mathrm{~nm}$. The model is a sum of exponentials with decay times $\tau_{\mathrm{i}}$, pre-exponential factors $\alpha_{\mathrm{i}}$ and fractional intensities $f_{\mathrm{i}}$

\begin{tabular}{lclll}
\hline & $\tau_{\mathrm{i}} / \mathrm{ns}$ & $\alpha_{\mathrm{i}}$ & $f_{\mathrm{i}}$ & $\chi_{R}{ }^{2}$ \\
\hline$\beta-\mathrm{CD}-\mathrm{PT} 7^{a}$ & $0.034 \pm 0.004$ & 0.496 & 0.17 & 1.04 \\
& $0.134 \pm 0.005$ & 0.489 & 0.65 & \\
& $0.66 \pm 0.12$ & 0.013 & 0.08 & \\
$\mathrm{Cd}^{2+} \subset \beta-\mathrm{CD}-\mathrm{PT} 7^{b}$ & $6.0 \pm 1.2$ & 0.002 & 0.10 & \\
& $0.037 \pm 0.010$ & 0.32 & 0.004 & 1.05 \\
& $1.51 \pm 0.17$ & 0.19 & 0.09 & \\
& $5.1 \pm 0.1$ & 0.41 & 0.63 & \\
& $11.7 \pm 0.3$ & 0.08 & 0.28 &
\end{tabular}

${ }^{a}$ Observation at $330 \mathrm{~nm}$. Channel width: 14.6 ps. $^{b}$ Observation at $400 \mathrm{~nm}$. Channel width: $24.4 \mathrm{ps}$.

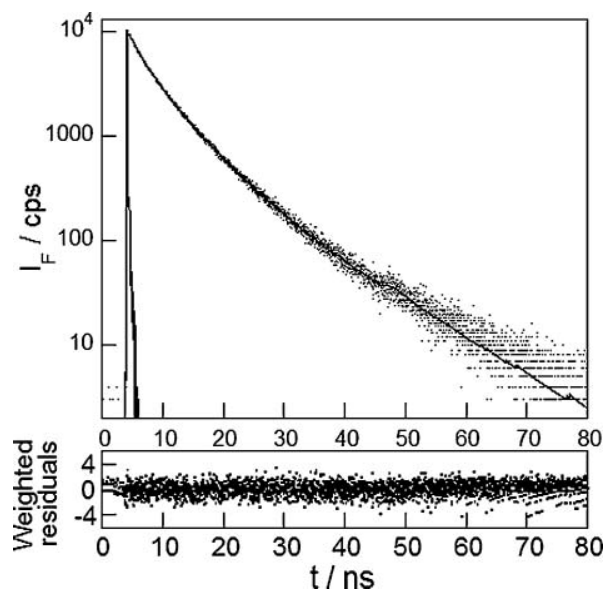

Fig. 4 Fluorescence decays of $\beta$-CD-PT7 $(1.7 \mu \mathrm{M})$ in methanol in the presence of one equivalent of $\mathrm{Cd}^{2+}$ Excitation wavelength: $288 \mathrm{~nm}$. Observation wavelength: $400 \mathrm{~nm}$. Channel width: $24.4 \mathrm{ps}$.

The results of the analysis with a sum of compressed hyperbola are reported in Table 5. It is remarkable that a single compressed hyperbola is sufficient to obtain a satisfactory fit of the decay in the absence of cadmium, whereas a sum of four exponential terms is necessary to get the same goodness of fit. The recovered distributions of decay times are shown in Fig. 5. The distribution is bimodal in the presence of cadmium. This could mean that there are two different populations of chromophores, but considering the complexity of the system and in the absence of evidence for excimer formation (in contrast to Calix-PT4), it is difficult to give an unambiguous interpretation. Nevertheless, it is worth noting that for both Calix-PT4 and $\beta$-CD-PT7, the long time distributions are characterized by an almost identical time constant $\tau$ (about $4.6 \mathrm{~ns}$ ), which however correspond to different average lifetimes ( $5.0 \mathrm{~ns}$ and $5.5 \mathrm{~ns}$, respectively) as the corresponding $c$ parameters are not identical.

\section{Conclusion}

The use of compressed hyperbola for recovering the underlying distributions in fluorescence decays was shown to be an efficient and reliable method. The small number of floating parameters used in the analysis makes us confident in the distributions recovered in the present study of multi-chromophoric systems. For instance,

Table 5 Data analysis of the fluorescence decays of $\beta$-CD-PT7 $(1,7 \mu \mathrm{M})$ in methanol in the absence and in the presence of one equivalent of cadmium. Excitation wavelength: $288 \mathrm{~nm}$. The model is a sum of compressed hyperbola according to eqn (3). $x_{\mathrm{i}}$ are the fractional intensities defined as: $x_{i}=\frac{a_{i} \tau_{i} /\left(1-c_{i}\right)}{\sum_{i} a_{i} \tau_{i} /\left(1-c_{i}\right)}$

\begin{tabular}{llllll}
\hline & $\tau_{\mathrm{i}} / \mathrm{ns}$ & $c_{\mathrm{i}}$ & $a_{\mathrm{i}}$ & $x_{\mathrm{i}}$ & $\chi_{R}{ }^{2}$ \\
\hline$\beta-\mathrm{CD}-\mathrm{PT7} 7^{a}$ & 0.077 & 0.26 & - & - & 1.09 \\
$\mathrm{Cd}^{2+} \subset \beta-\mathrm{CD}-\mathrm{PT} 7^{b}$ & 4.59 & 0.17 & 0.76 & 0.93 & 1.09 \\
& 0.52 & 0.60 & 0.24 & 0.07 &
\end{tabular}

${ }^{a}$ Observation at $330 \mathrm{~nm}$. Channel width : $14.6 \mathrm{ps}^{b}$ Observation at $400 \mathrm{~nm}$. Channel width : $24.4 \mathrm{ps}$. 


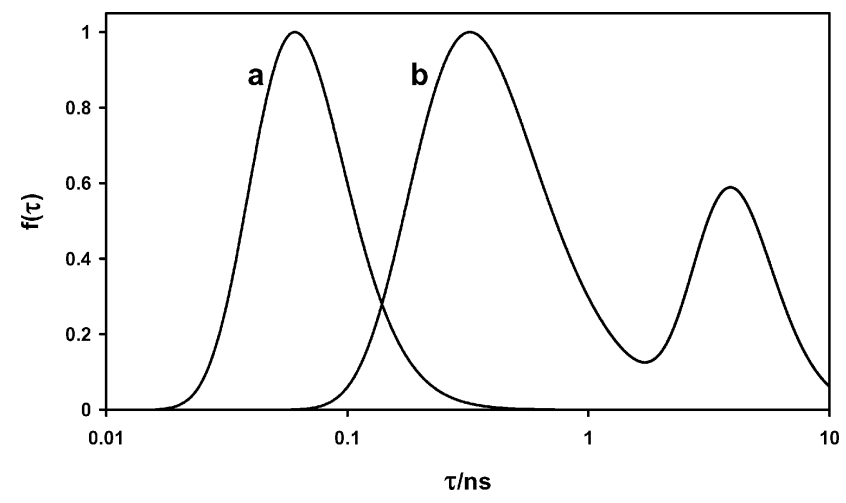

Fig. 5 Recovered distributions of decay times for CD-PT7 $(1.7 \mu \mathrm{M})$ in methanol in the absence (a) and in the presence of one equivalent of cadmium (b). For displaying purposes, each distribution was normalized at the respective maximum.

a single compressed hyperbola (i.e. with two floating parameters) was found to be sufficient to get a satisfactory fit of the fluorescence decay of an heptachromophoric cyclodextrin, whereas using the classical sum of exponentials, four terms were necessary (i.e. with seven floating parameters), and no physical significance can be assigned to the pre-exponential factors and time constants. The recovered distributions provide information on the heterogeneity of the micro-environments experienced by the chromophores, and/or on their mutual interactions in the excited state.

\section{Experimental}

\section{Materials}

The synthesis of Calix-PT4 ${ }^{7}$ and $\beta$-CD-PT7 ${ }^{8}$ were previously reported. Methanol from Aldrich (spectrometric grade) was employed as solvent. Cadmium perchlorate was purchased from Aldrich or Alfa Aesar and vacuum dried over $\mathrm{P}_{2} \mathrm{O}_{5}$ prior to use.

\section{Methods}

Fluorescence intensity decays were obtained by the single-photon timing method with picosecond laser excitation by use of a Spectra-Physics setup composed of a titanium sapphire Tsunami laser pumped by an argon ion laser, a pulse detector, and doubling (LBO) and tripling (BBO) crystals. Light pulses were selected by optoacoustic crystals at a repetition rate of $4 \mathrm{MHz}$. Fluorescence photons were detected through a monochromator by means of a Hamamatsu MCP R3809U photomultiplier, connected to a constant-fraction discriminator. The time-to-amplitude converter was purchased from Tennelec. Data analysis with a sum of exponentials was performed by a nonlinear least-squares method with the aid of Globals software (Globals Unlimited, University of Illinois at Urbana-Champaign, Laboratory of Fluorescence Dynamics). Data analysis with compressed hyperbola was achieved by means of a Microsoft Excel spread sheet specially designed for lifetime and anisotropy analysis (M. N. Berberan-Santos, unpublished).

\section{Acknowledgements}

The authors are indebted to Prof. Juan Xie for supplying a sample of $\beta$-CD-PT7 and Gluco-PT. MNBS was supported by FCT (Portugal) and POCI 2010 (FEDER) within research contract PTDC/ENR/64909/2006.

\section{References}

1 (a) L. Jullien, J. Canceill, B. Valeur, E. Bardez and J.-M. Lehn, Angew. Chem., Int. Ed. Engl., 1994, 33, 2438-2439; (b) L. Jullien, J. Canceill, B. Valeur, E. Bardez, J. P. Lefèvre, J.-M. Lehn, V. Marchi-Artzner and R. Pansu, J. Am. Chem. Soc., 1996, 118, 5432-5442; (c) D. M. Gravett and J. E. Guillet, J. Am. Chem. Soc., 1993, 115, 5970-5974; (d) N. Chattopadhyay, B. Haldar, A. Mallick and S. Sengupta, Tetrahedron Lett., 2005, 46, 3089-3092.

2 (a) B. Valeur and I. Leray, Inorg. Chim. Acta, 2007, 360, 765-774; (b) J. S. Kim and D. T. Quang, Chem. Rev., 2007, 107, 3780-3799.

3 (a) V. Balzani, F. Scandola, Supramolecular Photochemistry, Horwood, New York, 1990; (b) Molecular Switches, ed. B. L. Feringa, Wiley-VCH, New York, 2001; (c) V. Balzani, M. Venturi and A. Credi, Molecular Machines and Devices, Wiley-VCH, Weinheim, 2003; A. P. de Silva, N. McClenaghan, in Encyclopedia of Nanoscience and Nanotechnology, ed. J. A. Schwartz, C. Contescu and K. Putyera, Dekker, New York, 2004, pp. 2749-2756.

4 (a) M. N. Berberan-Santos and B. Valeur, J. Lumin., 2007, 126, 263-272; (b) M. N. Berberan-Santos, E. N. Bodunov and B. Valeur, Chem. Phys., 2005, 315, 171-182; (c) M. N. Berberan-Santos, E. N. Bodunov and B. Valeur, Chem. Phys., 2005, 317, 57-62.

5 (a) A. K. Livesey and J. C. Brochon, Biophys. J., 1987, 52, 693; (b) J. C. Brochon, Methods Enzymol., 1994, 240, 262.

6 A. Siemarczuk, B. D. Wagner and W. R. Ware, J. Phys. Chem., 1990, 94, 1661.

7 V. Souchon, S. Maisonneuve, O. David, I. Leray, J. Xie and B. Valeur, Photochem. Photobiol. Sci., 2008, 7, 1323-1331.

8 O. David, S. Maisonneuve and J. Xie, Tetrahedron Lett., 2007, 48, 6527 6530. 